\title{
Inherited disorders of straight chain fatty acid oxidation
}

The first inherited disorder of organic acid metabolism was described in 1966 , but it is only with the improvement in laboratory facilities in recent years that the true incidence of these disorders is beginning to be appreciated. Defects in the mitochondrial oxidation of straight chain fatty acids seem to be particularly common (as inborn errors of metabolism go) even though the first example, medium chain acyl-CoA dehydrogenase deficiency, was not properly characterised until 1982 . That such a major group of disorders should appear on the scene so late testifies to difficulties in recognition and investigation that still remain major problems.

\section{Biochemical background}

The presently known inherited disorders of mitochondrial oxidation of straight chain fatty acids all affect the acyl-CoA dehydrogenase step. Three different mitochondrial enzymes (abbreviated to LCAD, MCAD, and SCAD, respectively) catalyse the dehydrogenation of long chain, medium chain, and short chain acyl-CoAs. Deficiencies of each of these enzymes individually have now been recognised, though SCAD deficiency is rare, clinically distinct from the other types, and excluded from the following discussion. In addition, disorders of the mitochondrial electron transfer chain give rise to a state of multiple dehydrogenation deficiency. A mild form, often known as ethylmalonic-adipic aciduria (EMA), can resemble MCAD deficiency clinically and in the pattern of urinary metabolites.

Some patients seem to have disorders of fatty acid oxidation that do not correspond to any of those so far defined, and we may expect deficiencies of other enzymes in the mitochondrial $\beta$ oxidation pathway to be characterised within the next few years.

\section{Clinical presentations}

In all these disorders the inability to oxidise fats efficiently produces a tendency to fasting hypoglycaemia with inappropriately low ketonaemia accompanied, in severe attacks, by various secondary metabolic disturbances. The age of presentation is variable. LCAD deficiency is generally the earliest with three of the four patients so far fully described presenting soon after birth with hypoglycaemia, recurrent vomiting, liver dysfunction, cardiomegaly, and a tendency to cardiac arrest. ${ }^{1}$ MCAD deficiency is more common and generally less severe. It usually presents after 6 months of age, maybe even in the teens, often with a Reye like syndrome after a viral illness. Some children with MCAD deficiency may have repeated attacks of hypoglycaemia under minimal stress but others are completely asymptomatic in the absence of severe precipitating factors, even though a sib has died of the disease. EMA, too, may present with an acute Reye like attack, but our limited experience suggests that this disorder tends also to produce chronic problems with failure to thrive. ${ }^{2}$

\section{Diagnosis}

The first step is to collect urine samples during an acute attack. Timing is vital as the urine may appear completely normal between crises. The samples should be sent to an experienced laboratory for examination of urinary acids by gas chromatography, preferably combined with mass spectrometry. The free monocarboxylic acids are not excreted in any quantity but a rich variety of secondary products, ${ }^{3}$ particularly the even chain dicarboxylic acids, should be present during crisis in all these conditions. A rather similar dicarboxylic aciduria results from the administration of feeds containing medium chain triglycerides-for example, Pregestimil, Nenatal ${ }^{4}$-and it is always helpful (as well as being a matter of courtesy) to supply the investigating laboratory with brief clinical details and a full account of medication and feeds immediately before the collection of the specimen.

Sometimes the possibility of an acyl-CoA dehydrogenase deficiency may only be considered retrospectively after the attack or studies on asymptomatic sibs may be required, particularly in MCAD deficiency. In these cases it is usually possible to produce a diagnostic organic acid profile by fasting the patient, but this procedure has proved fatal and should only be undertaken with extreme caution. It may be necessary to prolong the fast well beyond 24 hours to be certain of a negative result. Fat loading is also intrinsically dangerous and should never be used. A safer approach using carnitine loading has recently been suggested ${ }^{5}$ but requires extremely specialised laboratory facilities. These problems may be circumvented by assaying the enzymes 
themselves, in cultured fibroblasts or in leucocytes. Methods suitable for large scale clinical application have recently been developed. ${ }^{6}$ They will find increasing application as they become available routinely as it is often difficult to distinguish with certainty between the different types of defect on the basis of urinary organic acid profiles alone.

\section{Treatment}

During an acute attack the immediate aim is to restore the blood glucose concentration. On thus relieving the pressure to metabolise fatty acids many of the secondary biochemical problems will resolve automatically but, in severe cases with Reye like features, measures to deal with cerebral oedema and electrolyte imbalance may be necessary.

In deciding long term management it can be helpful to assess the patient's susceptibility to hypoglycaemia during the overnight fast. If this is pronounced, as it is likely to be in LCAD deficiency, frequent carbohydrate rich meals are indicated. Excessive fat intake should be avoided in all these conditions. Valproic acid should not be given as it is likely to be unusually toxic to these patients.

Refractory cases may need additional measures. Some patients with EMA improve markedly on large doses of riboflavin. In LCAD deficiency correction of secondary carnitine deficiency may be helpful, though this treatment may be harmful in EMA. Medium chain triglycerides may be substituted for normal dietary fat in LCAD deficiency, but this could be fatal in MCAD deficiency. The diagnosis must be absolutely secure before these supplementary treatments are tried and it could be wise to consult an expert for the most up to date views in this rapidly developing field.

Many patients with MCAD deficiency are completely asymptomatic for long periods, indeed some may never show any sign of the abnormality, and it is important not to overreact to the diagnosis in a child who has had an isolated attack of hypoglycaemia. Appropriate advice on the dangers of prolonged fasting together with family studies to recognise other affected siblings are all that may be required.

MCAD and LCAD deficiencies can be diagnosed prenatally using cultured amniotic fluid cells should this be necessary.

\section{The size of the problem}

In the short time since the discovery of these conditions the number of cases known has increased rapidly. They seem to account for a small proportion of sudden infant deaths ${ }^{7}$ and a somewhat larger proportion of cases of Reye's syndrome. MCAD deficiency, in its milder presentations, is probably often overlooked. Isolated attacks of hypoglycaemia at home are likely to be labelled 'fits' without their metabolic basis being appreciated, and febrile convulsions too may be indicative of MCAD deficiency. Full investigation of all patients with the less specific indications is quite beyond the capacity of current laboratory facilities. Some form of preliminary screening, particularly a consistent and determined approach to the detection of intermittent hypoglycaemia, would produce a more manageable group for detailed investigation and should identify at least those patients most at risk of a severe attack.

\section{References}

${ }^{1}$ Hale DE, Batshaw ML, Coates PM et al. Long-chain acyl coenzyme A dehydrogenase deficiency: an inherited cause of non-ketotic hypoglycemia. Pediatr Res 1985;19:666-70.

2 Green A, Marshall TG, Bennett MJ, Grey RGF, Pollitt RJ. Riboflavin-responsive ethymalonic-adipic aciduria. J Inher Metab Dis 1985;8:67-70.

${ }^{3}$ Gregersen N, Kølvraa S, Rasmussen K, et al. General (mediumchain) acyl-CoA dehydrogenase deficiency (non-ketoic dicarboxylic aciduria): quantitative urinary excretion pattern of 23 biologically significant metabolites in 3 cases. Clin Chim Acta 1983;132:181-2.

4 Henderson MJ, Dear PRF. Dicarboxylic aciduria and medium chain triglyceride supplemented milk. Arch Dis Child 1986;61:610-1.

5 Roe CR, Millington DS, Maltby DA, Kinnebrew P. Recognition of medium-chain acyl-CoA dehydrogenase deficiency in asymptomatic siblings of children dying of sudden infant death or Reye-like syndromes. J Pediatr 1986;108:13-8.

${ }^{6}$ Frerman FE, Goodman SI. Fluorometric assay of acyl-CoA dehydrogenases in normal and mutant human fibroblasts. Biochem Med 1985;33:38-44.

${ }^{7}$ Howat AJ, Bennett MJ, Variend S, Shaw L, Engel PC. Defects of metabolism of fatty acids in the sudden infant death syndrome. Br Med J 1985;290:1771-3.

R J PollitT

Neonatal Screening Laboratory, Middlewood Hospital, Sheffield S6 1TP 